\title{
Tangence
}

\section{Une scénographie onirique}

\section{Les rêveries de la fermme sauvage d'Hélène Cixous}

\section{A Dreamscape}

\section{Les rêveries de la femme sauvage by Hélène Cixous}

\section{Isabelle Décarie}

Numéro 76, automne 2004

Figures de l'étrangeté. Proust, Musil, Pessoa, Cixous, Houellebecq

URI : https://id.erudit.org/iderudit/011217ar

DOI : https://doi.org/10.7202/011217ar

Aller au sommaire du numéro

Éditeur(s)

Tangence

ISSN

1189-4563 (imprimé)

1710-0305 (numérique)

Découvrir la revue

Citer cet article

Décarie, I. (2004). Une scénographie onirique : Les rêveries de la femme sauvage d’Hélène Cixous. Tangence, (76), 69-85. https://doi.org/10.7202/011217ar

\section{Résumé de l'article}

Hélène Cixous, dans son récit intitulé Les rêveries de la femme sauvage, emprunte à la psychanalyse le phantasme des origines et la scène primitive (rêvée, imaginée, inventée) afin de reformuler les souvenirs douloureux de son enfance passée en Algérie. Cette lecture se penche sur les stratégies d'écriture qui composent une scénographie onirique, laquelle se manifeste à travers plusieurs scènes marquantes qui tournent autour de l'identité sexuelle de la narratrice. La résistance au récit, l'oubli, l'aveuglement devant le passé sont autant de motifs qui sont analysés ici afin de mettre au jour une poétique de la confession où l'écriture, loin d'être réparatrice, est envisagée comme une rêverie, seule voie possible pour énoncer les trahisons de l'enfance. 


\title{
Une scénographie onirique: Les rêveries de la femme sauvage d'Hélène Cixous
}

\author{
Isabelle Décarie, \\ CÉLAT, Université du Québec à Montréal
}

\begin{abstract}
Hélène Cixous, dans son récit intitulé Les rêveries de la femme sauvage, emprunte à la psychanalyse le phantasme des origines et la scène primitive (rêvée, imaginée, inventée) afin de reformuler les souvenirs douloureux de son enfance passée en Algérie. Cette lecture se penche sur les stratégies d'écriture qui composent une scénographie onirique, laquelle se manifeste à travers plusieurs scènes marquantes qui tournent autour de l'identité sexuelle de la narratrice. La résistance au récit, l'oubli, l'aveuglement devant le passé sont autant de motifs qui sont analysés ici afin de mettre au jour une poétique de la confession où l'écriture, loin d'être réparatrice, est envisagée comme une rêverie, seule voie possible pour énoncer les trahisons de l'enfance.
\end{abstract}

Alors que certains récits d'Hélène Cixous se classent sous la dénomination quelque peu obscure de "Fiction", se distinguant ainsi de ses autres ouvrages qui portent l'appellation «Roman», "Essai » ou "Théâtre », on pourrait penser que l'expression "Scènes primitives", le sous-titre du récit dont il sera question ici, Les rêveries de la femme sauvage ${ }^{1}$, désigne un nouveau genre hybride dans la catégorie déjà protéiforme de l'écriture de soi. Plus encore, en faisant ouvertement référence à la scène originelle freudienne, le titre secondaire annonce l'exploration d'un passé reconfiguré par le phantasme des origines, la différence des sexes et des générations, ainsi que par la sexualité. On peut ainsi parler de

1. Hélène Cixous, Les rêveries de la femme sauvage. Scènes primitives, Paris, Galilée, coll. "Lignes fictives», 2000. Désormais, les références à cet ouvrage seront indiquées par le sigle $R S$, suivi de la page, et placées entre parenthèses dans le corps du texte. 
trucages de l'autobiographie où l'étrangeté prend le pas sur le réel. La scène primitive autant que la rêverie renvoient dans ce contexte à l'idée de fiction, de mise en scène de faits vécus à travers le filtre de l'écriture. Dès lors, il faut sans doute renvoyer au sujet qui s'articule dans ce texte par les termes "la narratrice» ou encore par «Hélène Cixous $^{2}$ » entre guillemets parce que le récit, à la manière de la cure psychanalytique, recrée les souvenirs à partir de l'écriture du présent et fausse ainsi toute perception univoque du sujet autographe. Il ne s'agit pas ici de relancer le débat en affirmant que le sujet ne s'élabore que dans et par l'écriture, mais bien plutôt de rappeler qu'il faut maintenir l'écart indécidable qui sépare l'auto du bio et de la graphie. C'est au cœur de ces intervalles indéterminés entre sujet, vie et écriture que se compose la scénographie onirique de Cixous, que s'écrivent les scènes marquantes, singulières et inquiétantes qui nous requerront dans les pages suivantes. Nous verrons dans un premier temps comment l'écrivaine décline les modalités du récit de soi et de la confession pour les transformer, puis nous nous pencherons sur les rapports entretenus entre chacune des scènes analysées et les figures parentales auxquelles elles se rattachent, et ce tout en montrant une attention particulière à l'écriture qui, loin d'être réparatrice, transcrit par renonciation les trahisons de l'enfance.

\section{Les partages d'un genre}

Divers motifs reliés pour la plupart au sexuel ont déjà fait l'objet d'une longue élaboration dans l'œuvre de Cixous, mais ils trouvent, avec Les rêveries de la femme sauvage, une expression plus singulièrement autogéographique, pourrait-on dire, où le lieu d'origine, l'Algérie, «ce pays natal inconnu» (RS, p. 167), devient le prétexte à une reconstruction narrative des événements marquants de l'enfance. La scène originelle, telle qu'elle fut élaborée par Freud, est ici déplacée du côté du primitif tellurique, où la terre d'origine, une «nébuleuse algérienne sexuelle» (RS, p. 24), encore à l'état sauvage de l'oubli, devient le personnage de ce songe éveillé, entre Oran la féminine et Alger la masculine. Mais le sol originel désigne aussi le terreau parental et particulièrement maternel (dont témoigne aussi le titre "Vues sur ma terre ${ }^{3}$ ", un texte publié par Cixous la même année et qui porte sur la figure de la

2. Tout comme le fait aussi Mireille Calle-Gruber pour parler de Derrida dans Marie-Louise Mallet (sous la dir. de), L'animal autobiographique. Autour de Jacques Derrida, Paris, Galilée, coll. «La philosophie en effet», 1999, p. 96. 
mère), sol retourné depuis lequel sont exhumés les aveux, les désirs blasphématoires, les blessures et les traumatismes. Ces scénographies s'engagent ainsi dans un double commencement puisque le titre est emprunté à une autre autobiographie qui marque le début d'une tradition du récit de soi selon la critique et son propre auteur, Jean-Jacques Rousseau. Si le titre du récit de l'écrivaine s'inspire surtout des Rêveries d'un promeneur solitaire auquel Les rêveries font allusion à deux reprises, on ne peut s'empêcher de penser aux Confessions de Rousseau dans lesquelles l'écrivain assure au lecteur, rappelons-le, qu'il «forme une entreprise qui n'eut jamais d'exemple et dont l'exécution n'aura point d'imitateur. Je veux montrer à mes semblables, poursuit-il, un homme dans toute la vérité de la nature; et cet homme ce sera moi ${ }^{4}$ ». Ce projet, qui a eu de multiples influences sur l'œuvre de Cixous, se démarque pourtant de celui des Rêveries de la femme sauvage, puisque ces dernières n'entretiennent pas la prétention de l'exemplaire ni de l'unique. Plutôt, elles donnent à penser, d'abord par le pluriel de leur dénomination, la multitude de possibilités pour raconter une vie. En d'autres termes, si les scènes mémorables demeurent définies, leur narration peut se décliner de maintes façons, faisant intervenir une multitude de codes qui brouillent toute appartenance générique stable. Jacques Derrida propose un partage qui permet de mieux apprécier encore ce qui se joue du point de vue du genre dans ce texte de Cixous. Il affirme en effet que «L'autobiographie devient confession quand le discours sur soi ne dissocie pas la vérité de l'aveu, donc de la faute, du mal et des maux ${ }^{5}$.» De la confession, du mal et des maux, il est bien question dans ce texte aux accents religieux, où la venue à l'écriture dans les toutes premières pages ressemble à une véritable liturgie dans laquelle se côtoient les annonciations, les auréoles et «l'hostie qui répand chair et sang du Venant» $(R S, \mathrm{p} .10)$. Ce récit loge ainsi dans un lieu flou entre la profession de foi (on voit là l'influence des Confessions rousseauistes dont le destinataire est bien Dieu) et le phantasme, s'inscrivant de la même façon dans une appétence pour le tragique familial (CEdipe n'est pas loin).

3. Hélène Cixous, «Vues sur ma terre», dans Mireille Calle-Gruber (sous la dir. de), Hélène Cixous, croisées d'une xuvre, Paris, Galilée, coll. «La philosophie en effet", 2000, p. 235-254.

4. Jean-Jacques Rousseau, Les confessions, Livre premier, Paris, GarnierFlammarion, 1968, p. 43.

5. Jacques Derrida, "L'animal que donc je suis (À suivre)", L'animal autobiographique, ouvr. cité, p. 272. 
Dans l'entretien Photos de racines, dont le titre met en avant encore le géologique, paru en 1994, Cixous confirme à son interlocutrice qu'elle ne croit pas à l'autobiographie, mais plutôt à la confession: «Il peut y avoir les fractures du moi passionnantes que sont les confessions. Ce sont pour moi des œuvres, des livres. Appelons cela autobiographie, mais c'est une version. C'est la version de l'aveugle ${ }^{6}$.» Dans cette perspective, la confession serait donc une variante aveugle et aveuglante de l'autobiographie, une déclinaison qui permettrait les interprétations dues à la cécité, puisque dans l'obscurité la fiction, comme une prothèse, compense pour ce qui ne peut être vu. Il s'agit là d'un motif important dans l'œuvre de l'écrivaine car elle parle ouvertement de sa myopie paralysante dont elle a aussi raconté l'opération corrective dans "Savoir ${ }^{7} »$. Selon elle, ce handicap aura été déterminant dans sa manière d'appréhender le monde: les événements sont racontés dans le détail (comme un oil se collant au plus près de la chose à décrire) ou encore inventés parce que trop flous. Cixous offre ainsi de manière paradoxale une confession faite de rêveries dont le point aveugle constitue aussi le pacte entre le texte et son lecteur. En effet, le vrai et le faux ne représentent plus des notions vérifiables: ils ont été remplacés par les soupçons du lecteur.

Que la confession s'effectue sur un divan ou dans un confessionnal, elle s'exécute toujours dans la cécité parce qu'elle n'a pas à souffrir le regard de l'autre. La confession s'aveugle ainsi d'une autre manière encore puisqu'elle fait fi des répercussions «du mal et des maux» qui seront proférés, comme ce fut le cas avec Osnabrück, un livre tout particulièrement cinglant à l'égard de la mère, et dans lequel le frère interroge justement la narratrice sur la réception maternelle du récit ${ }^{8}$. Les scènes primitives de Cixous ressemblent de la sorte à ces photographies factices et spectrales que déclenche la narratrice dans Les rêveries (nous y reviendrons); elles s'apparentent à une absence devenant réalité, à un phantasme devenant vérité, à une fiction devenant une archive, pour reprendre les termes d'une expression semblable de Jacques

6. Hélène Cixous et Mireille Calle-Gruber, Photos de racines, Paris, Éditions des Femmes, 1994, p. 96.

7. Hélène Cixous, "Savoir », dans Hélène Cixous et Jacques Derrida, Voiles, Paris, Galilée, coll. "Incises», 1998, p. 11-19. Désormais, les références à cet ouvrage seront indiquées par le sigle $S$, suivi de la page, et placées entre parenthèses dans le corps du texte.

8. Hélène Cixous, Osnabrück, Paris, Éditions des Femmes, 1999. 
Derrida $^{9}$. Les rêveries le suggèrent d'ailleurs de manière différente lorsque la narratrice écrit: «il m'apparaît aujourd'hui que nous étions déjà dans un livre sans le savoir » $(R S, \mathrm{p} .72)$.

Dès lors, plus que la mémoire, c'est la question de l'oubli et de la résistance au récit qui modèle ce texte, où le lecteur assiste, dans les premières pages, à la naissance du livre venu de la nuit, à l'écriture aveugle des premières lignes «à travers le flot abondant des rêves» (RS, p. 10), «dans une joie sans garde» (RS, p. 11), où les notations oniriques produites sur le coup de la levée de l'oubli seront remplacées par la perte du manuscrit:

"Tout le temps où je vivais en Algérie je rêvais d'arriver un jour en Algérie, j'aurais fait n'importe quoi pour y arriver», avais-je écrit [...]. Des cinq pages que j'avais écrites dans une joie sans garde, et que je n'ai pas inventées car je les ai vues écrites, je ne trouvais plus que la demi-feuille, la première, sur laquelle sans même allumer $\mathrm{j}$ 'avais écrit les lignes "Tout le temps où je vivais en Algérie, etc. ( $(R S$, p. 9 et p. 11, souligné dans le texte).

L'Algérie enfin ressouvenue se serait donc évanouie avec le jour; la suspension de la censure aura été trop violente pour se rendre à l'écriture: voilà du moins ce que la narratrice tente de faire accepter au lecteur, lequel ne peut s'empêcher de trouver la coïncidence presque trop belle pour être vraie. S'agirait-il ici d'un faux oubli, d'un acte manqué factice ou tout simplement d'un de ces rêves si translucides qu'on ne peut distinguer la réalité du songe? Plutôt, cette scène laisse penser que le désir d'oublier les années algériennes se fait pressant, mais que ce souhait rencontre une obligation insistante de se rendre d'abord au récit pour être précisément enfoui, comme s'il fallait soumettre les souvenirs tenaces (parce que douloureux) aux griffes acérées de l'écriture afin d'entretenir l'illusion qu'on les aura rayés de la mémoire. Alors que la plupart des auto(bio)graphes écrivent pour se souvenir, ici, on peut penser que Cixous entre dans cette écriture aveuglée pour enfin laisser derrière elle ces années d'apprentissage, où elle tente d'anéantir par l'écriture son propre être algérien, à la manière de cette Algérie française dont elle parle.

La fin du texte laisse bien entrevoir une autre logique de l'autographie: "Je n'ai jamais voulu écrire sur l'Algérie ce pays

9. Jacques Derrida, "Lettres sur un aveugle. Punctum cacum», dans Jacques Derrida et Safaa Fathy, Tourner les mots. Au bord d'un film, Paris, Galilée/Arte Éditions, 2000, p. 78. 
natal inconnu dont j'ai longé la haute blancheur fermée pendant tant d'années, l'idée même d'écrire ne me serait même jamais venue» (RS, p. 168). Leitmotiv qui ponctue nombre de ses récits, la résistance à l'écriture, en s'inscrivant tout de même dans le filigrane du texte, participe de la même économie que l'oubli transformé en souvenir par le biais du récit. Or, comme Cixous le remarque elle-même, le travail de mémoire effectué sur ce pays de l'Unheimliche (le familier inconnu, le natal étranger) a commencé dès 1994 avec Photos de racines et suit une trajectoire assez découpée, avec la parution du livre du père (Or: les lettres de mon père) et celle d'Osnabrück, puis des Rêveries et aussi avec la publication du Jour où je n'étais pas là qui retrace un événement grave, la mort d'un fils trisomique en $1961^{10}$. La narratrice des Rêveries rapportera d'ailleurs ces paroles de son frère furieux parce que leur mère leur a offert un seul vélo à partager, un vélo de femme (nous y reviendrons):

- Rien ne devient souvenir jamais, dit mon frère dans le fauteuil. Il n'y a pas d'oubli. Regarde ce Vélo regarde. Il n'y a pas de mémoire. C'est un crime dit mon frère. C'est une incompétence maternelle totale dit le pédiatre. C'est la même toujours il n'y a pas de temps. Tandis que pour moi pensai-je tout ce qui entre en criant sur la scène sort sur la scène de papier $(R S$, p. 36).

Il n'y a certes pas de temps pour le traumatisme comme nous l'a appris Freud, puisque l'inconscient ne connaît pas de temporalité vectorielle. Tout laisse penser que l'écriture ici temporalise l'événement bouleversant relié au vélo, comme c'est le cas aussi pour les autres scènes troublantes qui composent le livre. La scénographie de la narratrice permet, en quelque sorte, une séparation et une mise à distance grâce à ce cadre inventé par l'écriture. Pour elle, même s'il n'y a pas non plus d'oubli, la scène est tout de même transformée en récit et, de ce fait, la fiction remplace alors l'oubli. La perte de mémoire n'existe pas car le deuil de cette époque résiste: la narratrice emploie un terme évocateur pour décrire cet état des choses en suspens, en attente d'être refoulées. À propos de la perte des fragments écrits la nuit, elle remarque:

10. Hélène Cixous, Or: les lettres de mon père, Paris, Éditions des Femmes, 1997; Hélène Cixous, Le jour où je n'étais pas là, Paris, Éditions Galilée, coll. «Lignes fictives», 2000. 
Cela ressemble tellement à cette sorte de maladie algérie que je faisais en Algérie ou qu'elle me faisait, cette sensation d'être possédée par une sensation de dépossession et la réponse que je produisais, ce combat pour conquérir l'introuvable qui peut me conduire à l'autodestruction, tout comme autrefois, ici, dans mon bureau, après si longtemps. En proie à l'indeuillable mon âme se remord jusqu'aux sangs ( $R S$, p. 17, souligné dans le texte).

L'indeuillable décrit bien la blessure dont on ne peut se séparer, mais surtout la persistance et la pérennité de la lésion dont on ne saurait se détacher. Dès lors, ces Rêveries affichent une tentative de réactualiser cette possession/dépossession afin de la détruire par l'écriture. Toutefois, on sait combien le deuil de soi résiste à toute écriture dite de la réparation. Plutôt, le récit compose un nouveau cadre dans lequel quelque chose de cette résistance à l'oubli trouvera tout de même à se dire à travers le filtre et le déguisement de l'histoire officielle, celle de l'Algérie française. Cette contrée composite et singulière tente, selon la narratrice, de mettre en place un "programme initial secret [qui] est un plan d'effacement de l'être algérien, qui s'accomplissait exactement comme tous les plans d'effacement semblables en vigueur dans tous les pays qui appliquent la substitution totale. Substitution, ablation, et fantomatisation, des opérations effectuées avec un succès total» $(R S, \mathrm{p} .124)$. En déplaçant ce programme sur la scène du texte, la substitution, l'ablation, et la fantomatisation deviennent dès lors les motifs, ainsi que les opérations textuelles et narratives qui définissent la tentative d'autodestruction à l'œuvre.

\section{Se séparer d'une séparation}

À la manière d'un préambule au Bildungsroman intellectuel qu'il n'écrira jamais et qui s'écrit pourtant dans le filigrane de chacun de ses textes, Jacques Derrida, dans Le monolinguisme de l'autre, explique le rôle qu'a joué la langue française dans son apprentissage de la philosophie, langue qu'il parle et dans laquelle il écrit, mais qui n'aura jamais été sienne, tout à fait. Le français demeure pour lui une langue maternelle qui ne lui appartient pas, une langue maternelle étrangère, dont la caractéristique contradictoire provient de son lieu de naissance et de son pays natal, l'Algérie. Le philosophe remarque d'ailleurs, à propos d'une phrase d'Hanna Arendt sur la langue allemande, que la relation à 
la mère et à la langue maternelle «nous reconduit vers la grande question du phantasme, de l'imagination comme phantasia et lieu du phantasma ${ }^{11}$ ». Les rêveries sont elles aussi le théâtre de ces rapports complexes entre langue et famille. Comme le souligne encore Derrida, Hélène Cixous écrit à la croisée de deux langues: «Cette grande-écrivain-française-juive-d'Algérie-sépharade qui réinvente, entre autres, la langue de son père, sa langue française, une langue française inouïe, il faut rappeler que c'est aussi une juive-ashkénaze-allemande par la "langue maternelle" 12 ». Ainsi les scènes primitives montrent-elles le clivage instauré entre le désir relié au père, le passeur de la langue française maternelle que Cixous situe du côté de la littérature, et la mère dont la langue est étrangère à la famille. On peut penser que ce désir d'arriver dans ce pays natal étranger qu'est l'Algérie, où la mère ne souffre d'aucun exil parce qu'elle réussit à reconstruire son nid "sans jamais tomber malade, et surtout pas d'amour» (RS, p. 58), traduit aussi le phantasme de vouloir se séparer de cette séparation inaugurale - une mère tranchante qui ne ressent rien - qui aura toujours déjà été présente sous la forme d'une figure tout entière vouée à la coupure, sous toutes ses formes. La question posée en quatrième de couverture des Rêveries, "Comment se séparer?", pourrait donc aussi vouloir dire de manière plus troublante encore: comment se séparer d'une séparation? Comment l'écriture, qui est inscription, peut-elle tenir compte de l'absence, de la coupure et de la perte? En 1971, après un éloignement de près de vingt ans, la narratrice retourne sur les lieux de son enfance où les portes de la maison ont été remplacées par des murs. Elle se rappelle alors cette parole de sa mère: "Tu dois rompre la poche des eaux, dit ma mère, sinon c'est la fin pour l'enfant dans la mère avec la mère. J'ai dû pleurer» $(R S$, p. 166).

Ainsi, entrer en Algérie, cela revient peut-être aussi à partir en coupant le cordon, à reprendre par l'écriture le métier de la mère (elle était accoucheuse) et procéder soi-même à l'ablation de quelques lignes écrites dans la nuit. Mais la langue maternelle «se parle» aussi à travers les gestes que fait la mère, ces agissements parfois singuliers et incisifs qui sont tout aussi éloquents. "Traumatisme inaugural» $(R S$, p. 25$)$ pour le frère, la scène du vélo met en avant la question de la différence sexuelle depuis l'angle de la

11. Jacques Derrida, Le monolinguisme de l'autre, Paris, Éditions Galilée, coll. «Incises», 1996, p. 105. L'auteur souligne.

12. Jacques Derrida, Le monolinguisme de l'autre, ouvr. cité, p. 114. 
mère qui offre à ses enfants une bicyclette allemande féminine. À travers le récit de la narratrice, on comprend que l'absence de cette barre qui différencie le sexe du vélo a l'effet d'une castration pour son double masculin, le frère, lequel parcourra tout de même le corps/ravin de la Femme sauvage (c'est le nom d'un ravin non loin de la maison), malgré l'aveuglement de la mère pour sa masculinité. Destinée au jardin, à sa chambre et à ses livres parce qu'elle abandonne le vélo à son frère, la narratrice, pour sa part, remarque: "maman n'a même pas voulu me castrer» (RS, p. 37, souligné dans le texte). Pourtant, cette mère coupeuse de cordons (RS, p. 59) et dont la sœur (elle est dans la couture) a aussi appris la coupe, est bien du côté de la castration, de l'ablation: c'est par souci de coupures dans les dépenses qu'elle achète un seul vélo et de surcroît féminin pour ses deux enfants; elle est également une "amputée souffrant de ne pas souffrir» (RS, p. 57), selon les paroles du frère. Dans Osnabrück, le vocabulaire de la mutilation servait déjà à marquer la relation entre la fille et sa mère: "Elle part comme on oublie. [...] Elle me troue. Elle perfore. Et elle sort. Par le trou de mon ventre je suis tombée. [...] Je suis dans l'amputation ${ }^{13}$ ». De la même façon, dans Le jour où je n'étais pas là, on apprend que la mère suggère à sa fille de se faire refaire le nez:

Je venais d'avoir 14 ans, et je traduisis mon nez en chose à couper, en élément honteux, en pavillon de laideur flottant sur mon paysage $[. .$.$] . Nez coupé, terminé, le sang une fois répandu$ dans la poussière ne remonte pas dans les veines, ce nez-là, mon héritage, mon père, je ne veux pas m'en séparer, le spectre de mon père me hantait et ne disait pas un mot, ce qui condamnait à une difficile liberté. J'ai craint de me couper de mon père ${ }^{14}$.

En imposant un vélo allemand pour femme à ses deux enfants après la mort de son mari, et en proposant à sa fille de couper son nez paternel, la mère aura tenté de faire régner une loi tranchante et doublement maternelle (avec Omi, la grand-mère complice), afin d'éloigner sa fille de sa posture masculine et paternelle à Alger. L'être masculin au Clos-Salembier (le nom que porte la maison) aura donc été victime d'une ablation semblable à celle de l'être algérien. La transfiguration de cette coupure mortelle se fera sentir dans une scène importante qui marque d'un sceau tragique

13. Hélène Cixous, Osnabrück, ouvr. cité, p. 25.

14. Hélène Cixous, Le jour où je n'étais pas là, ouvr. cité, p. 59. 
l'enfance de la narratrice. Raconté bien après la scène du vélo dans le livre, mais antérieur dans la chronologie des événements, le passage "de la fille coupée en deux" se situe à Oran, la ville dans laquelle on entend les lettres centrales ( or») de Georges, le prénom du père ${ }^{15}$. La description de la ville permet de mieux saisir le drame du voile mortel:

[...] la différence entre Oran et Alger est sexuelle, Oran m'était femme et Alger l'homme, à Oran je faisais la femme, à Alger l'homme, à cause de la façon dont la Ville d'Oran était d'après moi toute séduction ronde rose épicée aux aisselles, toute fuite, je lui courais après, elle toutes voiles et voiles, mutine, vivante, évaporée, moi toujours égarée, perdue dans les vapeurs mauves du bain maure, amoureuse palpant les corps devenus infinis de mes parents dissous (RS, p. 49).

Ces dernières lignes donnent bien à lire une scène primitive à laquelle l'enfant participe au même titre que les parents et dans laquelle les deux types de voiles (la voile et le voile) renvoient à la différence sexuelle, à un autre partage du genre. Dans la scène de la fille coupée en deux, le port de cet «organe masculin» (RS, p. 102) qu'est le voile mène à la coupure ultime, celle de la mort. Enlacée par un homme, une jeune femme périt sous les roues d'un manège, entraînée par son voile. La spectatrice réécrit la scène de manière à montrer qu'il s'agit là de son tout premier souvenir "ayant trait au Plan d'anéantissement de l'être algérien» $(R S$, p. 144). S'identifiant à la jeune fille coupée en deux, la narratrice comprend dès lors l'impossibilité de ne jamais atteindre l'Algérie:

C'est une tragédie qui est aussi une Ville, un pays, une histoire, l'histoire de celle que je ne suis pas, un voile nous sépare et pour cette raison même je sens un voile tomber une buée rouge sur ma tête sur mes épaules [...] malgré moi je porte une jeune fille coupée en deux le voile mortel la coupure parce que je suis une fille témoin de la victime, coupée de la victime. [...] Depuis l'accident quelque chose en moi me reste voilé ( $R S$, p. 146).

Cette histoire primitive de voile natal, Cixous l'aura transposée dans son œil, dans le champ de la myopie et du "voile natal», cette fois celui dont il est question dans "Savoir", où elle raconte qu'elle est née le «voile dans l'œil» $(S$, p. 14): «La joie de l'œil délivré physiquement: une sensation délicieuse d'agrafes ôtées: car la myopie a de petites serres, elle tient l'œil sous un voile

15. Voir à ce sujet Jacques Derrida, «H. C. pour la vie, c'est à dire...», dans Hélène Cixous, croisées d'une cuvre, ouvr. cité, p. 29. 
serré, vissements de paupières, insistances, efforts vains pour passer le voile et voir, front froncé» $(S$, p. 18). Rattachée à la figure maternelle, la myopie enfin corrigée devra faire place à un deuil: "Soudain ma myopie, "l'autre", s'est dévoilée [...] Adieu ma mie ma mère» $(S, \mathrm{p} .17)$. Coupée elle aussi de son voile comme la jeune fille du manège, l'écrivaine espère enfin pouvoir faire le deuil de sa terre natale qu'est cette mère embrumée et déjà perdue à plusieurs reprises à cause de son aveuglement. Séparation métaphorique, la myopie, cette "étrangeté» $(S$, p. 16) enfin corrigée et expulsée, ne fera pourtant rien de plus pour colmater la brèche entre la mère et sa fille. Dans «Vues sur ma terre", Cixous explique qu'un matin, alors qu'elle prenait sa voiture, elle aperçut de l'autre côté du trottoir sa mère qui habite tout près, et s'assura d'être vue par elle. La mère finalement lui sourit. L'écrivaine raconte cette anecdote afin de montrer combien fut grande la joie d'apercevoir d'aussi loin sa mère. Le soir, Cixous lui demande si elle l'a bien vue, et la mère répond oui, sans soupçonner ce qui ébranle sa fille et elle ajoute: "J'ai pensé, lui dit-elle, que tu allais encore une fois chez le coiffeur ${ }^{16}$.» On le voit aisément, la mère coupeuse de cordons n'a bien que la coupure en tête, et quand il ne s'agit pas du nez de sa fille, il est question de ses cheveux. Ainsi, le plan d'anéantissement de l'être algérien désigne-t-il aussi l'ablation incessante instiguée par la mère pour transformer la fille, ce que l'écrivaine transpose à son tour par le truchement de l'écriture et à travers le récit de l'excision du «secret de son enfance» $(S$, p. 17) que fut sa quasi-cécité.

\section{Déguisements du récit}

Si cette histoire de fille coupée en deux est un des tout premiers souvenirs de l'écrivaine, elle rapporte pourtant que la blessure la plus vive provient d'une autre scène, cette fois reliée à la figure du père. Avant de venir à la scène de la poupée, disons quelques mots sur cet homme auquel Cixous a aussi consacré un livre. Dans Or: les lettres de mon père, qui précède la sortie du livre sur la mère, Cixous inscrit son père dans une filiation littéraire singulière dès l'ouverture du texte. En exergue, elle reproduit en effet une page du journal de Kafka où il est question de Georg, le propre père de l'écrivain tchèque: «J'étais avec mon père dans le

16. Hélène Cixous, «Vues sur ma terre», Hélène Cixous, croisées d'une æuvre, ouvr. cité, art. cité, p. 241. 
vestibule d'un immeuble; dehors, il pleuvait à verse. Un homme, venant de la rue, s'apprêtait à chercher refuge dans le vestibule, quand il aperçoit mon père. Il s'arrêta. "Georg", dit-il lentement, comme s'il avait à ramener graduellement de vieux souvenirs à la surface; et, en avançant la main, il approcha de côté vers mon père ${ }^{17}$.» Avec la publication en 2002 du récit Manhattan. Lettres de la préhistoire, le premier récit sur le père prend une tout autre ampleur, et l'on comprend de manière aiguë le rapport équivoque qui relie Cixous à Kafka et l'importance capitale de l'œuvre de ce dernier pour l'entrée de Cixous en littérature - pour une entrée dans ce que la littérature a de plus noir, de plus opaque, de plus fictif. Manhattan est le récit tragique d'une supercherie: dans les années 1960, la narratrice, qui vient de perdre son père Georges et son enfant trisomique, prénommé Georges aussi, rencontre dans une bibliothèque américaine où elle travaille à sa thèse sur Montaigne un jeune homme qui s'appelle Gregor, tout comme le personnage de La métamorphose, la nouvelle de Kafka. L'inconnu, qui tente de séduire la narratrice, se fait passer pour mourant et l'entraîne dans un jeu de mensonges et de lettres d'amour factices. La narratrice, aveuglée par le chagrin de la mort de ses deux Georges, attirée par ce faux malade qui partage l'initiale paternelle et filiale (cette lettre G qui est aussi celle de la préhistoire du soustitre) et par le caractère romanesque de ce qui lui arrive, reconnaîtra trop tard que les lettres reçues avaient été plagiées: Gregor lui a envoyé en son nom les lettres de Kafka à Milena. L'épistolaire, comme Jacques Derrida l'a longuement montré dans les «Envois» de La carte postale, ce texte lui aussi hanté par le nom camouflé de Kafka, spectralise l'autre, le destinataire de la lettre qu'on interpelle et invoque en son absence par l'écriture. Entre la lettre G et la correspondance de Kafka, le père et le fils sont revenus hanter la narratrice par le biais d'une littérature qui appartient d'emblée au régime de l'inquiétant. C'est aussi de cette façon que Cixous convoque son père dans "Vues sur ma terre», juste avant de consacrer la suite du texte à la mère, où elle raconte les «revenances» et retours périodiques de la figure paternelle disparue ${ }^{18}$. Dans Les rêveries, la fantomatisation s'intègre aussi à la mise à mal de l'être algérien qui se révèle tout particulièrement dans l'épisode de l'appareil photographique qui a appartenu au père:

17. Hélène Cixous, Or: les lettres de mon père, ouvr. cité, exergue.

18. Voir Hélène Cixous, «Vues sur ma terre», art. cité. 
Moi aussi je suis initiée au camouflage, au déguisement, au semblant, à la feinte, au masque. [...] J'en vins à la fantomatisation. Moi-même, je suis devenue mon fantôme [...] La meilleure façon pensai-je d'être une forteresse imprenable c'est de se retirer derrière les apparences. Sur quoi je pris l'appareil de photo irréparable de mon père que ma mère avait jeté aux ordures mais que j'avais ramassé quoiqu'irréparable. Et j'en fis un outil fantomatisant ( $R S$, p. 149).

L'artifice des photographies transforme ainsi le réel en apparition, geste qui n'est pas étranger au phantasme lui-même, lequel reconfigure la réalité selon le désir. Tout comme on a pu constater une résistance au deuil, c'est-à-dire de l'indeuillable, au début du récit avec la mère, ici l'irréparable (un état défectueux, cassé, déclaré par la mère) est récupéré afin de parer mécaniquement, artificiellement, à l'absence du père. Remarquons au passage le nombre de termes dont les préfixes traduisent le négatif, comme ici «indeuillable», « irréparable», mais aussi «imprenable», «l'appareil inhabileté»; et dès les premières pages du récit: «le surgissement espéré mais inimaginable du livre» $(R S$, p. 9), "la précipitation de ces phrases inestimables» $(R S, \mathrm{p} .10)$, "l'idée de reconstituer les pages était inacceptable» $(R S, \mathrm{p} .12)$, «la ville d'Alger me restait intouchable» (RS, p. 13), « une maladie antimaternelle» $(R S$, p. 27$)$, "la disparition irréversible de notre père» $(R S$, p. 28$)$, etc. Ces expressions fortes accentuent la question de la résistance au récit, comme si les préfixes négatifs donnaient à lire un barrage de la lettre par la lettre et rappelaient au lecteur que la narratrice s'adonne à l'écriture par renonciation.

Si l'appareil photographique permet de substituer une réalité à une apparition, l'épisode de la poupée met en place une économie textuelle semblable qui relève tout particulièrement du travail du rêve. Après avoir raconté qu'elle doit se rendre à un concert avec son amie Françoise et le rêve engendré par cette activité à venir (quels vêtements doit-on porter pour aller au concert?), la narratrice écrit :

Mais tout cela n'est que divertissement à mon plus ancien deuil. C'est l'ouvrage de mon père. C'est le coup. C'est la blessure. L'événement, le mien. L'ouverture à l'œuvre, ce que mon père m'a fait, une fois, la seule fois, une violence unique dont le père ne mesure pas l'interminable ricochet cruel, comment regretter la douleur? elle m'est d'une infinie fidélité ( $R S$, p. 133).

Alors que la narratrice a neuf ans, elle voit de la fenêtre de la Citroën familiale, une poupée mauresque dans une vitrine et elle 
demande à son père de la lui acheter, ce qu'il refuse. Il s'agit d'un passage décrivant l'incision, la blessure primitive à l'origine de l'œuvre à venir :

Il est le roi. Il ne voit rien [...] Je vois tout. Je sais tout. Le Voile me dit tout. [...] Il ne me voit pas. Il croit que je suis un enfant. Il croit qu'il est un père. [...] C'est comme si je commettais un parricide dans la Citroën. Je le sais. Je le commets. Dans l'auto je ne suis pas l'enfant. Il y a substitution. [...] Je suis adultère. J'entre dans la minutie de la passion je veux tout et je veux chaque partie je veux le fin voile de visage, je veux le haïk de lin et de soie, je veux l'agrafe d'argent [...] je veux être le Saroual je veux l'Algérie. [...] Comment te pardonner? Tout le reste est déguisement (RS, p. 134-135) ${ }^{19}$.

On l'a déjà dit, le voile interdit reviendra dans l'œuvre de Cixous à maintes reprises: c'est le voile dans l'œil qui travestit la réalité, c'est le voile mortel de la jeune femme du manège (à laquelle renvoie aussi la poupée), c'est le voile dont Cixous se sert pour déguiser sa propre écriture souvent régie par la figure de la métalepse. L'ouvrage du père, c'est donc à la fois ce tissu mortel et blessant fait de fibres crues et soyeuses, de lin et de soie, mais c'est aussi l'événement qui fonde le Livre (tous les livres) de Cixous. C'est l'ouvrage dans lequel il faut entendre l'outrage. Le refus du père engendre un récit d'extrême blessure parce qu'il aura refusé de convoiter sa propre fille, laquelle s'identifie à la poupée dédaignée. L'idée du parricide, mais aussi le vocabulaire du tragique dans le récit entier, laisse entrevoir une mise en scène de séduction blessée. En effet, juste avant ce passage, la narratrice raconte divers rêves qu'elle aurait faits avant d'aller au concert avec son amie Françoise. Cette dernière représente, et déjà par son prénom, la France catholique en Algérie. La narratrice se demande quels vêtements elle pourra porter à cette occasion, ce qui déclenche chez elle des rêves de déguisements et de costumes. Le tout dernier qui est raconté est celui dans lequel elle tente de vendre le collier d'or de sa grand-mère pour s'acheter des habits neufs. Mais les Arabes qui passent devant elle détournent le regard et ne veulent pas de son bijou. Au lieu d'écrire «personne ne veut

19. Ici encore, la figure de Kafka se profile derrière cette promesse et je pense plus particulièrement à Lettre au père (Franz Kafka, Lettre au père, traduit de l'allemand par Marthe Robert, Paris, Gallimard, coll. "Folio», 1957). Voir à ce sujet Jacques Derrida, Donner la mort, Paris, Éditions Galilée, coll. «Incises", 1999, p. 182. 
acheter mon collier", elle écrit plutôt: "Personne ne veut m'acheter» (RS, p. 133). Dès lors, le récit laisse entendre, par l'emboîtement narratif et la juxtaposition du rêve et de la scène de la poupée, que le père, à la manière des Algériens qui ne veulent pas de l'or juif, ne désire pas acheter la poupée mauresque à laquelle s'identifie l'enfant, qu'il ne veut donc pas de sa fille accoutrée de cette façon, sexualisée et "arabisée ».

\section{Une mémoire sonore}

Si la blessure du père est d'une "infinie fidélité» — l'allitération ici redouble l'importance du syntagme -, on peut rapprocher cet épisode de celui de la morsure du chien, ce troisième "enfant» de couleur fauve qui partage avec le père les mêmes tonalités dorées. Cadeau du père à ses enfants (en lieu et place d'un frère ou d'une sœur), le chien cristallise aussi un rapport œedipien transposé dans le couple que la sœur forme avec son frère. Créant en effet une gémellité troublante et pour le moins incestueuse sur le plan du phantasme, le frère et la sœur ont pourtant un rapport différent au chien, car ce chien deviendra le modèle des souffrances de la narratrice. L'intérêt de cet épisode réside sans doute dans le terme "inséparables" qu'elle emploie pour décrire l'accident de la morsure. Alors qu'on sonne à la porte du ClosSalembier, la narratrice se lève de sa lecture et pose un pied devant le chien surpris qui riposte avec les dents: "De cette morsure je vais mourir, car elle ne me lâche plus elle s'enfonce sans fin et pénètre jusqu'à mon cœur, nous entrons dans l'éternité de la folie, pensai-je, la tête environnée de nuées écarlates. Les dents duraient. Nous étions devenus inséparables ${ }^{20} »(R S$, p. 80$)$. Cette union incorporée par la souffrance décrit aussi le lien que la narratrice entretient avec l'Algérie, son "algie», sa "maladie algérie» (RS, p. 16, souligné dans le texte) qu'elle transpose dans le néologisme «inséparabe» $(R S$, p. 89). À la manière des Algériens qui subissent une substitution violente, celle de leur terre natale devenue étrangère par la force de la loi française, la narratrice endure une même contradiction fondamentale où le chien domestiqué (le compagnon soi-disant fidèle) et aimé a fait place à une bête inconnue qui, d'un bond, a personnifié sa couleur fauve. Exilée en terre

20. Voir à ce sujet le très beau livre d'Hélène Cixous, L'amour du loup et autres remords, Paris, Éditions Galilée, coll. «Lignes fictives», 2003. 
devenue primitive par ce don du père, la narratrice se rend donc à la logique de la traîtrise en déplaçant l'Unheimliche territorial sur la scène de l'écriture, à travers le récit de cette figure inaugurale canine qui aura engendré ces rêveries de la femme devenue sauvage par la trahison: «[...] c'est le destin du Chien selon moi qui est la métaphore et le cœur de toute l'histoire de nos Algéries [...] Mon âme Le Chien. Ma transfigure sauvage» (RS, p. 73).

Partageant donc avec le chien un destin d'enfermement derrière les barreaux de la grille (elle a abandonné le vélo à son frère), et en même temps se sentant exilée, étrangère dans son propre pays natal, la narratrice entre en littérature par le biais de ce chien qui est d'abord du côté de la lecture et ensuite de l'écriture. Les toutes dernières pages des Rêveries rappellent comment ce livre a vu le jour - ou, plus justement, la nuit. Entre le désir de ne jamais écrire sur l'Algérie et l'insistance d'une trace mémorielle sonore, la narratrice évoque, à la manière du narrateur proustien qui entend encore tinter dans les dernières pages de la Recherche l'ovale doré de la clochette du jardin, le souvenir audible de la voix du chien (et remarquons au passage que c'est bien la sonnette du jardin qui aura transformé dans les Rêveries le chien en bête sauvage):

Le souvenir d'une voix. La trace sonore de la voix de Fips quarante ans après sa mort. Je ne pouvais plus désormais chasser le livre qui ne cessait de m'appeler dès que j'ouvrais la fenêtre de l'obscurité. Je me suis redressée dans mon lit en pleine nuit et avec le crayon gras qui est toujours couché à côté de ma main j'ai écrit à grands traits dans le noir: Tout le temps où je vivais en Algérie je rêvais d'arriver un jour en Algérie (RS, p. 168, souligné dans le texte).

L'image des toutes dernières lignes du récit accentue la scénographie déployée autour de cette figure du manuscrit rêvé/perdu dont le livre serait la retranscription «de mémoire». En somme, la narratrice a reconstitué ici des souvenirs découpés et agencés en autant de scènes inaugurales, primitives; des événements qui ont été rassemblés autour de figures et d'objets (le Vélo, le Chien, la Poupée, le Manège) qui ont recomposé les années algériennes de la petite enfance selon le paradigme de la terreur, de la souffrance, de la trahison.

Le souvenir doit-il toujours être inquiétant, celui d'une blessure, pour se rendre à l'écriture? Est-ce parce que la mémoire agit comme un garde-fou que les souvenirs les plus incisifs tardent à se 
livrer? Plutôt que de se souvenir, il s'agit au contraire, pour Cixous, de rêver. Les rêveries permettent de se rendre au livre grâce aux transfigurations, aux déplacements, aux substitutions, aux variations de l'écriture noire de la nuit; une écriture qui déplace le retour en Algérie sur la scène onirique et recomposée d'un temps perdu - un temps perdu qui doit à tout prix le rester. 